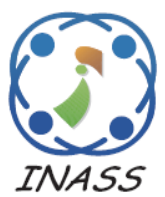

\title{
EPBDRA: Efficient Priority Based Dynamic Resource Allocation in Heterogeneous MIMO Cognitive Radio Networks
}

\author{
Tamilarasan Santhamurthy ${ }^{1 *}$ \\ Kumar Parasuraman ${ }^{1}$ \\ ${ }^{1}$ Centre for Information Technology and Engineering, Tirunelveli, Tamilnadu, India \\ * Corresponding author's Email: stamilarasan74@ rediffmail.com
}

\begin{abstract}
The cognitive radio (CR) offers keen solution to the serious scarcity of spectrum in wireless communication. In Multi-Input and Multi-Output (MIMO), primary user recruits some secondary users to cooperatively relay the primary traffic. It is very difficult to assign channel allocation and resource distribution in heterogeneous networks. We propose EPBDRA-CRN technique which provides priority scheduling scheme resulting in more reliability and efficient data transmission. The resource allocation can be done dynamically after ensuring that the nodes satisfy the specified constraints such as fairness, minimum-rate-guarantee, and minimum-delayguarantee. Proposed EPBDRA-CRN algorithm does resource allocation and scheduling for PU and SU to exploit the performance of entire network by reducing the interference at PU. The proposal also supports different rates of transmission with priority, minimizes the transmission delay and improves fairness and provides significant network throughput and avoids collision. We compare EPBDRA-CRN with existing DRA-CRN algorithm using ns-2 simulation. The simulation result shows that the proposal is $27 \%$ more efficient than the existing DRA-CRN algorithm.
\end{abstract}

Keywords: Cognitive radio, MIMO, SINR, Dynamic allocation, OFDMA.

\section{Introduction}

$\mathrm{CR}$ is an emerging technology which gives a suitable solution for the future generation of wireless networks. The wireless communication system has a lack of spectrum utilization. CR is a promising technology and provides a smart solution to improve the spectrum utilization. To avoid scarcity of wireless spectrum, researchers have designed intelligent secondary network concepts which can enable opportunistic access of spectrum free from licensed users or primary users (PU) [1]. $\mathrm{CR}$ is an intelligent wireless communication system that utilizes spectrum opportunistically by monitoring the licensed frequency spectrum to reliably detect PU signals and operate the secondary users (SU) whenever the PU is absent [2]. The detection of PU signals as characterized by spectrum sensing, geolocation database usages or combination of both. The functionalities of cognitive radio are spectrum access, cooperative communication method and reducing the interference and these technologies are deployed in various networks like MANET, WLAN, broadcasting television networks etc. [3].

MIMO is a new technology developed in the physical layer and it could have many advantages since it uses many antennas and follows enhanced signal processing techniques. MIMO operates the utilization of altered transmitter and getting receiving wires at the presented hubs to form the usage of system ideally and to operative multipath spread for expanding dependability $[2,3]$.

In this paper, we can mainly focus on increasing the capability of the secondary network during the exploration of the MIMO-CRN. The dynamic resource allocation technique maximizes the spectrum utilization, power allocation for multichannel, selection of secondary users based on their QoS requirements [4]. The advantages of CRMIMO are largely independent and parallel [6]. 
In this paper, we propose an efficient priority based dynamic resource allocation in MIMO cognitive radio network (EPBDRA-CRN). The priority queue scheduling algorithm is adapted with SBS. This algorithm controls transmission between primary and secondary networks and provides a significant throughput improvement than existing DRA-CRN transmission algorithm. The proposed algorithm supports different priorities, reduces the interferences at $\mathrm{PU}$, and reduces the transmission delay in the given network.

MIMO-CRN technology has more advantages like independent and parallel working nature. MIMO-OFDM technology is a hybrid of both MIMO and OFDM (orthogonal frequency division multiplexing) features. MIMO technology improves the multiple utilization capacity by transmitting different signals over multiple antennas [4, 6]. OFDM technique splits a radio channel into a huge quality of closely spaced sub-channels to offer more reliable communication at extraordinary speed [8]. MIMO-OFDM is smart technology of choice for next generation wireless systems such as IEEE802.11n, IEEE802.16, and the future generation cellular [9]. This technology has better performance and bit error rate (BER) in any given network of operation [12].

The proposed EPBDRA-CRN algorithm provides the benefits of CR and MIMO by achieving an overall higher throughput and lower delay in heterogeneous wireless networks. In the rest of this article we present literature survey in section II, problem identification and solution in section III, the proposed solution in section IV, simulation result in section $\mathrm{V}$ and conclusion of this article in section VI.

\section{Literature survey}

Senthilmurguan.S [1] presented predictive channel selection algorithm to allocate resource in multichannel CRN.

S. Chu [2] presented holistic scheduling algorithm which opportunistically schedules the resource to transmit and select the available channels in distributed fashion with respect to channel information and also proposed centralized and distribution algorithm to achieve network performance.

S.J. Kim [3] presented two algorithms namely convergent distributed algorithm without CR constraints which provide optimal solution locally and semi-distributed algorithm with CR constrains which provides optimal solution during iterative procedure implementation.
D.W.K. Ng [4] presented the dynamic resource allocation with scheduling design for MIMOOFDMA system with full duplex and hybrid relaying as a non-convex and combinatorial optimization problem and they considered heterogeneous users and the loop-interference cancellation error. Further, they could have been developing an efficient iterative distributed resource allocation algorithm with closed form of power and subcarrier allocation policies based on dual decomposition approach to solve the problem. A. Ghosh [5] presented cross layer antenna selection algorithm to improve the MIMO transmission efficiency and learning based algorithm which moderates the data rate variance among cognitive nodes as well as number of channel switching events.

$X$. Xiao [6] primarily focuses on energyefficient resource allocation in a MIMO-OFDMA system based on LTE standard. They have formulated the energy efficiency algorithm using mathematical equivalence to perform joint sub channel assignment and power allocation with Lagrange dual method. They improved the energy efficiency of the resource allocation using an efficient algorithm based on the integration of bisection search and ellipsoid method.

Y. An [7] presented two algorithms for improving the performance of cognitive radio wireless local area network based on cooperative transmitting approach. This system does not have priority scheduling approach.

M. Ghamar [8] presented an adaptive transmission strategy for underlay MIMO cooperative cognitive radio networks.

Z. Khan [10] presented a simple adaptive winshift lose-randomize strategy and also have utilized the framework of repeated games with imperfect observations and limited memory for dynamic resource allocation but it does not use priority scheduling strategy.

D.T. Hoang [11] presented an online learning algorithm that observes the environment and adapts the channel access action accordingly without any prior knowledge about the model parameters. The algorithm involves a Markov decision process to achieve the optimized solution to improve throughput.

S. Tapaswi [15] presented a novel algorithm to model the dynamic channel allocation problem in cognitive radio networks. The proposed system contained unique system for dynamic channel allocation but no specific approach has been considered for priority scheduling.

W. Wang [16] presented distributed scheme or optimal resource allocation without exchanging 
spectrum dynamics information between remote nodes. They used queue-balanced system for resource allocation in the network. Node based distributed algorithm is proposed to allocate power between nodes.

A. Mohamedou [17] presented centralized and distributed algorithm for power distribution between multiple nodes.

M. Khasawneh [20] presented power trading approach with Nash equilibrium algorithm which is implemented at SU for improving power during transmission.

P. Reba [21] presented an efficient channel selection algorithm at transmitter and receiver side which will select multiple antennas for data transmission.

S. Tamilarasan [26, 27] presented dynamic resource allocation with priority scheduling for heterogeneous cognitive radio network which supports an effective utilization of channels when PU is idle but this algorithm doesn't used in MIMO cognitive radio networks.

\section{Problem identification and solution}

From this literature survey, it can be concluded that there is no work which jointly provides dynamic resource allocation and priority scheduling approach in MIMO heterogeneous cognitive radio network. We consider that heterogeneous cooperative CRN services would be difficult in allocating the resource effectively and hence design a channel allocation to each node in the available network. SUs with different rates are considered and the delay requirements are not considered [12].Further, we use the channel quality indicator (CQI) for estimation of channel quality while transmitting the resources in the given network [22, 23].

We propose to develop a dynamic resource allocation with priority scheduling for MIMO heterogeneous cooperative CRN. The network contains primary users (PUs) and secondary users (SUs) by different service requirements in a MIMO Cooperative CRN. Secondary network (SN) contains SUs and secondary base station (SBS), the SBS is responsible for assigning resources to each node in the given network. The primary network (PN) contains PUs and primary base station (PBS). SU should have different characteristics such as minimum rate guarantee with SUs (MGR-SU), minimum delay guarantee with SUs (MDG-SU), minimum rate and delay guarantee with SUs (MRDG-SU) and best effort service with SUs (BES$\mathrm{SU})$. During the resource allocation to the nodes the following constraints should be met:
- Total power restriction [12] which includes the total transmitted power of SU on all channels must be within the power budget at the SBS.

- For MRG-SU, the transmission rate for SU should be greater than the minimum-rate threshold [12].

- For MDG-SU, the transmission delay for SU should be less than the deadline threshold [12].

- For MRDG-SU, the transmission rate should be greater than the minimum-rate threshold and the transmission delay should be less than the deadline threshold [12].

- For BES-SU, fairness constraint should be satisfied [12].

During the transmission, we can measure the channel quality by using a utility function like CQI $[22,23]$. The CQI is estimated in terms of the signal-to-interference-plus-noise ratio (SINR) [24], [25]. We have introduced priority queue scheduling algorithm which measures the priority of each incoming packet streams. The packets priority is measured depending on the service type and queuing delays [12].

CQI is estimated for each incoming packets stream by multiplying the priority with channel gain. Then, the assigned priority stream values are stored in descending order. Then we can assign the values to the respective type of SU. The highest objectives of the packet stream are allocated to the MRDG-SU, followed by MGR and MDG. The packet stream with least objective function is assigned to BES-SU $[12,13]$.

\section{Proposed solution}

\subsection{System model}

The CRN consists of PN and SN. The PN consist of PUs (Licensed users) and PBS. The SN consists of SUs (Unlicensed users) and SBS. Both PN and SN use OFDM technologies. The SN should sense the unused primary channels and opportunistically exploit the idle channels for heterogeneous services by using time-slice system. SN senses $M$ channels in each time slice intervals and effectively utilizes the idle channels when PU is idle. Channels are sensed by using channel sensing technology which is adopted at secondary network. These techniques monitor the channel status and sense the channel and communicate the sensing information to the SBS in SN. Then the SBS should make decisions to define whether the idle channels are available or not. The priority scheduling algorithm is implemented at SBS in SN $[12,13]$ 
Whenever the PUs does not access the channels, at that time the SUs should access the idle channel optimally. Periodically, the occurrence of a PU is checked for and if found the channel is returned to the PU. This prevents SU interference with PUs and the idle channels are accessed only when PU is not accessing the assigned channel [3, 12].

PUs operations are given more priority during the channel exploitation and PU should not be disturbed by any interference like satellite communication, medical equipment's. SBS controls the resource allocation for SUs in the given network. According to SUs request SBS would determine which channel to access and which SU to be provided the channel. In this article, we consider the following characteristics of MIMO heterogeneous SUs.

- K1: Minimum-rate guarantee with SUs (MRG$\mathrm{SU})$

- K2: Best effort service with SUs (BES-SU)

- K3:Minimum delay guarantee with SUs (MDG$\mathrm{SU})$

- K4: Minimum rate and delay guarantee with SUs (MRDG-SU)

We assume that $\mathrm{N}$ idle sub-channels are in the given time slots and SBS senses these $\mathrm{N}$ idle channels $(N \leq M)$. The access of $N$ channels varies dynamically in different time slice intervals. The channel state is determined for $M$ channels by comparing total power $\left(\mathrm{POW}_{\mathrm{m}}{ }^{\text {TOTAL }}\right)$ assigned to $\mathrm{M}$ channels with threshold value $\left(\mathrm{TE}_{\mathrm{m}}\right)$ [12].

In this paper, we mainly focus on power, channel and resource allocation. Also to reduce the complexity of the network, we assume $K 1, K 2, K 3$, and $K 4$ relation as $K 1=K 2=K 3=K 4$. The $\mathrm{SN}$ has equal number of heterogeneous SUs with MRG-SU, MDG-SU, MDRG-SU and BES-SU. We assume that during the resource allocation, the SBS allocates these K SUs on N channels. The objective of the channel selection system is to give the channel information. Based on the channel state, and the resource allocation time, data is transmitted between SUs and SBS in the data transmission time $[12,13]$.

The SBS will perform optimal resource allocation to heterogeneous SUs and PUs with the following assumption.

- Let each and every channel be assigned to one SU. The binary index method $\eta k, n \in\{1,0\}$ is used to represent channel allocation. If ( $\eta n, k$ $=1$ ) then channel $\mathrm{n}$ is allocated to SUk. Otherwise channel $\mathrm{n}$ is not allocated to SUk.

- Total power constraint: Let $P O W_{\text {TOTAL }}$ be used to denote the total power budget required for each SUs in the given network. Here $\rho k, \mathrm{n}$ is used to represent the transmit power for SUk in subchannel. In each time slot, the base station transmits a power for SUs in the network.

$$
\sum_{k=1}^{k 1+k 2+k 3+k 4} \sum_{n=1}^{N} \eta_{k, n}, \rho_{k, n} \leq \text { POW }
$$

- Minimum rate guarantee: The SUs transmission performance is determined by minimum rate threshold guarantee $\left(\mathrm{MR}_{\mathrm{k}}{ }^{\mathrm{min}}\right)$ in the given network.

$$
M R_{k} \geq M R_{k}^{\min } \forall k \in k 1 \cup k 2 \cup k 3 \cup k 4
$$

- Proportional-fairness constraint: The fairness guarantee for SUk is $k i$ or $\mathrm{k} 1$ or $k 2$ or $\mathrm{k} 3$ or $\mathrm{k} 4$. Assume that the proportional factor is $\delta_{k}$

$$
\frac{M R_{k}}{\sum_{i \in k 1 \cup k 2 \cup k 3 \cup k 4} M R_{i}}=\forall k \in k 1 \cup k 2 \cup k 3 \cup k 4
$$

Where $\delta_{k}, \quad \forall_{k} \in k 1 \cup k 2 \cup k 3 \cup k 4$ values are predetermined.

- During resource allocation, we consider bandwidth metrics, because the performance of the network depends on the bandwidth capacity.

$$
\begin{gathered}
M R_{k}=\sum_{n=1}^{N} \eta_{n, k} E_{T B} \log _{2}\left(\frac{1+1.5 \rho k, n S N R_{k, n}}{\ln \left(\frac{0.2}{B E R_{T A R G E T}}\right)}\right) \\
\forall k \in k l \cup k 2 \cup k 3 \cup k 4
\end{gathered}
$$

Where, $E_{T B}$ is a transmission bandwidth on each channel. $B E R_{\text {TARGET }}$ is target bit error rate. SNR is signal-to-noise ratio[12], [13].

\subsection{MIMO-CRN}

MIMO-CRN consists of $\mathrm{PN}$ and SN. PN consists of PUs and PBS. SN consists of SUs and SBS. Let us assume that there are $\mathrm{P}$ numbers of secondary user which is denoted by $\Psi P=\{1,2,3$ $\ldots P\}$ and $\mathrm{N}$ number of orthogonal channels denoted by $\Psi N=\{1,2,3 \ldots N\}[10]$.

In the secondary network, the SUs are well furnished with two MIMO antennas. The SBS is linked with each SUs and it is the SBS that will decide on which channel should be assigned to 
which SU for transmission. PUs are linked with SUs by $M$ channels. Let us assume that the successful transmission between $\mathrm{PU}_{\mathrm{TX}}$ and $\mathrm{PU}_{\mathrm{RX}}$ in the $\mathrm{PU}$ node during the concurrent transmission between $\mathrm{SU}_{\mathrm{TX}}$ and $\mathrm{SU}_{\mathrm{RX}}$ is well-established [7, 10, 12, 13, 18].

During the transmission, $\mathrm{SU}_{\mathrm{TX}}$ should have limited energy for transmission so that interference power received at primary receiver node $\mathrm{PU}_{\mathrm{RX}}$ will not go beyond its maximum threshold level. The presence of PU at the SBS should estimate channel quality periodically by using channel quality indicator (CQI). Whenever the value of CQI is decrease beyond the threshold level, it identifies the presence of existing PU. Then the SU reverts the sub-channel back to the PU. The total bandwidth of cell is B and each sub channels contains an equal quality of sub-carriers. Each sub channels bandwidth quality is calculated by $\mathrm{B} / \Psi_{\mathrm{N}}$. CQI of any sub-channel is defined using the Signal-ToInterference-Plus-Noise Ratio (SINR) detected by the SBS during channel allocation [14, 15, 24, 25].

$$
\operatorname{SINR}=\frac{P_{\text {SIGNAL }}}{P_{\text {INEREERENCE }}+\text { NOISE }}
$$

Where $P_{\text {SIGNAL }}$ is an incoming signal power, $P_{\text {INTERFERENCE }}$ is the background power, NOISE is some noise term. It may be constant or random. Assume that $P_{\text {INTERFERENCE }}=0$ then SNIR reduces signal to signal-to-noise-ratio (SNR). At SBS the channel quality indicator is measured by [19].

$$
C Q I=\log _{2}(1+\operatorname{SINR})
$$

Initially, the idle channels are determined by the PU and its information will be transmitted to SU (either $\mathrm{SU}_{\mathrm{TX}}$ or $\mathrm{SU}_{\mathrm{RX}}$ ). Here the SUs sense the available channels and determine the idle channels in the network. The base station in the network should be deciding the status of the channels. The priority queue system is adopted with the SBS. The secondary user should make channel access by picking one of the available channels in the networks. Here we can determine the priority of each incoming packet streams by CQI status and delay of queuing system. Then the objective function is estimated for each and every incoming packet stream by multiplying the priority with channel gain. If the SU can select an idle channel then it will start data transmission. During the data transmission SU can retrieve data packets from the queue $[12,15]$. The secondary user requires power for data transmission in each time slots [20]. The estimated priority values are stored in descending order and then the priority values are assigned to the specific SU. The packet stream with the highest priority objective function is allocated to the MRDG-SU followed by MRG-SU and MDG-SU. The stream with least priority objective function is assigned to BES-SU.

\subsection{Channel allocation for SU and handoff}

When data transmission takes place, all nodes in heterogeneous CRN are intermediate nodes. Here data transmission takes place between PUs and SUs. Nodes which are likely to be close to $\mathrm{PU}_{\mathrm{RX}}$ are called $\mathrm{SU}_{\mathrm{RX}}$ and nodes that are likely to be close to $\mathrm{PU}_{\mathrm{TX}}$ are called $\mathrm{SU}_{\mathrm{TX}}$. We can assume that the total numbers of SUs in SN are SUTOTAL. The SN is well equipped with nodes and MIMO antennas. Here all SN consists of SUs and SU-BS. The SU-BS takes decision for channel allocation to the SUs based on its priority. The SU generates its request and transmits the request to SU-BS. The SU-BS receives the request signal from the $\mathrm{SU}$ and assigns the priority value to each incoming request. The priority values are stored in descending order in the priority queue. The PU is communicated with the SU by M channels.

The CQI continuously monitors and estimates the channel quality. Once the value goes less than the threshold value, the channel handoff to the PU in PN takes place.

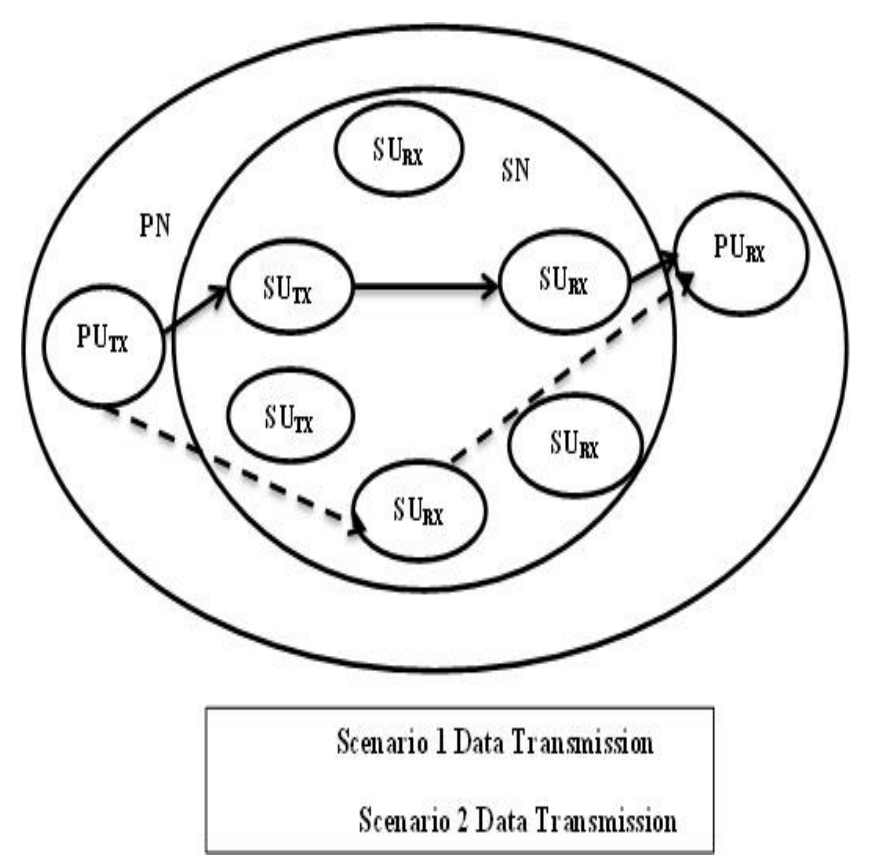

Figure.1 MIMO- CRN data transmission 


\subsection{Algorithm for channel allocation}

Step 1: $\quad$ Define sub-channel $K 1$ and $K 2$

Step 2: Data transmission takes place between $P U\left(P U_{T X}\right.$ and $\left.P U_{R X}\right)$ and $S U$ ( $S U_{T X}$ and $S U_{R X}$ ).

Step 3: Each sub-channel works on FrDiv (Frequency Division) system by using TDMA technique.

Step 4: SU senses the $M$ channel to identify available idle channel.

Step 5: Sensing information transmit to SBS

Step 6: SBS total power with threshold.

Step 7: If(Sensing Information Power > Threshold|HO) then

- Select idle channel (HO)

- Calculate false alarm probability of $\mathrm{M}$ channels.

$$
P_{m}^{F A}=P_{r o b}\left\{P O W_{m}^{\text {TOTAL }} \geq_{T E} \mid H o\right\}
$$

- Request Terminated

Step 8: If(Sensing Information Power > Threshold|H1) then

- Select busy channel (HI)

- Calculate false alarm probability of $\mathrm{M}$ channels.

$$
P_{m}^{D E T}=\operatorname{Prob}\left\{P O W_{m}^{\text {TOTAL }} \geq T E_{n} \mid H_{l}\right\}
$$

- Request Terminated

Step 9: If SBS senses $\mathrm{N}$ channels and assign time slots to each channels.

- If $(N \leq M)$ then

- The SBS is assigned SU with the specified time slots.

- Request Terminated, then GOTO step 4.

4.5 Algorithm for efficient priority based dynamic resource allocation (EPBDRA-CRN)

Step 1: Determine the sub-channels and assign $K 1$ and $K 2$

Step 2: $S U$ request to $S B S$ for sub-channel allocation.

Step 3: $S B S$ getting the sensing information and perform channel estimation.

Step 4: All altered nodes like $K \leq N$ and all $S U s$ in $S N$ network are accessed.

Step 5: $\quad S B S$ check the value of $\eta n, k$ and asks $P B S$ for sub-channel allocation.
- If $(\eta n, k=1)$ then, channel $N$ allocated to $S U k$, then request terminated

- Else, channel $N$ not allocated to $S U k$, then request terminated.

Step 6: $\quad$ Check $C Q I$ at $S B S$

- If (CQI $\ngtr$ Threshold) then,

- Request terminated.

Step 7: $\quad S U$ checks $M G R-S U$ constraints

- If ( $M R_{k} \geq M R_{k}^{\min }$ ) then,

- MGR-SU constraint is satisfied,

- Else $M G R-S U$ constraint is not satisfied, request terminated.

Step 8: Check Fairness at $S U$

- If $\left(\frac{M R_{k}}{\sum_{i \in k i} M R i}=\forall K i\right)$ then

- Fairness constraint satisfied,

- request terminated

Step 9: Check BES-SU constraint

- If ( $B E S-S U \geq T E)$ then,

- $B E S-S U$ constraint is satisfied

- Else, not satisfied

Step 10: Priority will assign to all $S U S$

Step 11: Checks the priority value at $S U s$ -

Neighbour nodes $\left(Q_{P R}^{T X}\right)$

Step 12: Check the neighbourhood transmitter constraints $T X$

- $T X=\{N i-B i\}$ if $2|b i|<\mid N i$, Then,

- A neighbourhood transmitter constraint $T X$ is satisfied. Else

- $T X=\{\lceil N i / 2\rceil\}$ Then

- A neighbourhood transmitter constraint $T X$ is not satisfied.

Step 13: If (Random Number $\leq Q T X$ ) then

- Rights to transmits. Else

- No Rights to transmit

Step 14: The highest priority node is selected from the database at SBS

Step 15: Frame Division (FrDiv) divided into initial FrDiv and final FrDiv

- $\quad(1-\lambda) F r D i v$

Step 16: During initial FrDiv

- $S U_{T X}$ is nominated by the $P U_{T X}$ is permitted to access the channel.

- Data transmission take place between $P U_{T X}$ and $S U_{T X}$, then 
forwarded to $S U_{R X}$, finally delivered to the $P U_{R X}$.

- Destination transmit $A C K$ message during the next FrDiv

Step 17: During final FrDiv

- $S U_{R X}$ nominated by the $P U_{T X}$ is permitted to access the channel

- Data transmission takes place between $P U_{T X}$ and $S U_{R X}$.

- $\mathrm{SU}_{\mathrm{RX}}$ forwards it directly to the PU $U_{R X}$.

- Destination transmit $A C K$ message during the next FrDiv.

Step 18: Update Priority, then

Step 19: Check the queue size

- If (Queue Size « Threshold) then,

- GOTO step 11

Step 20: Idle channel is allocated to $S U$ with the highest priority and least queue size

Step 21: Check transmitted power is less than total power allocated to the channel then data transfer takes place.

Step 22: Power allocation takes place for the $S U$ allotted with the sub-channel, then data transfer is take place

Step 23: Check channel quality indicator status at SBS.

- If (CQI $>$ threshold) then

- request terminated

- Else

- GOTO step 10.

\subsection{Existing algorithms (DRA-CRN)}

The existing algorithm provides the dynamic resource allocation in CRN. This algorithm was not hybrid with priority scheduling method as stated in the articles [10,12, 13, 17, 21].

Step 1: Define n sub-channels for $S U S$

Step 2: $\quad$ Sense the available $P U s$ and $S U s$

- If PU channel is sensed then

- Handoff to $S U$

- Else

Step 3: Sensing a free available channels

Step 4: $\quad$ Sense the available $P U s$

- If $P U$ channel is sensed then

- Handoff to $S U$ in $S N$

Step 5: Preferable channel list $(P C L)$ is used to allocate the available resource to SUs

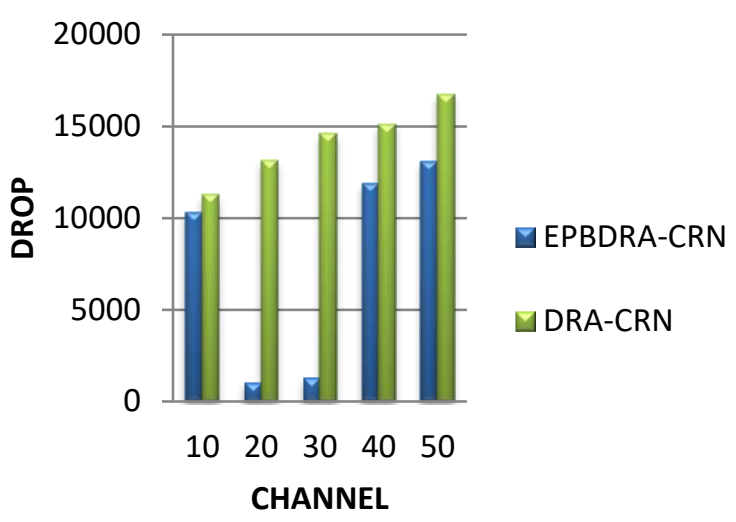

Figure.2 Channels vs. Drop

Step 6: Power allocation takes place for channel allocation to the give $S U$

Step 7: Data can take place transmission, then

Step 8: Sense the available channels

- If $P U$ channel is sensed then

- Handoff to the given $S U$

- Else

Step 9: Channels are assigned to other $S U S$

Step 10: $P C L$ assigned value to $S U$ based on its request order

Step 11: GOTO STEP 2

Step 12: Request Terminated

\section{Simulation results}

In CRN, dynamic resource allocation algorithm was used [10, 12, 13, 17, 21]. This algorithm does not provide priority scheduling methods. The proposed algorithm provides dynamic scheduling with priority. Here spectrum hand-off takes place between PU and SU. Each incoming packets priority is determined on CQI and queuing delay [13].

In Fig. 2, during the transmission, drop refers to the average number of packets dropped. Each channel in the CRN experiences drop with respect to the number of idle channels and mobility of nodes present in the network. In CRN, if idle channels are increased from 20 to 30 channels, the packet drop due to the employment of proposed system is found to be negligible as compared to existing system by $47 \%$. Due to prioritization of SU at the SBS, each idle channel in the network experiences less delay and more throughputs. From Fig. 6, it can be observed that proposed system is found to experience $19 \%$ of fewer drops in data packets in comparison to existing system. 


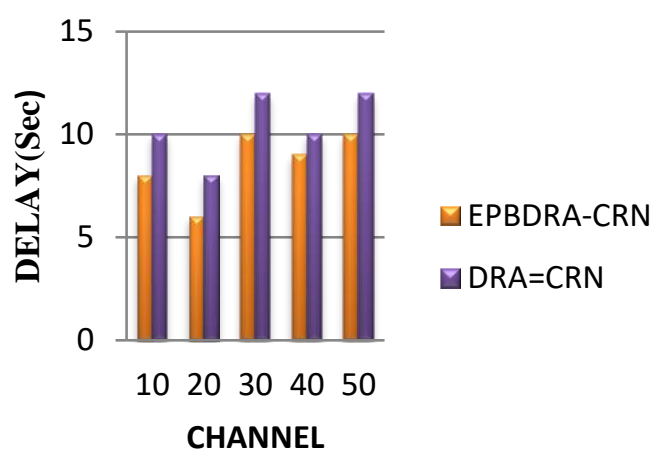

Figure.3 Channels vs. Delay

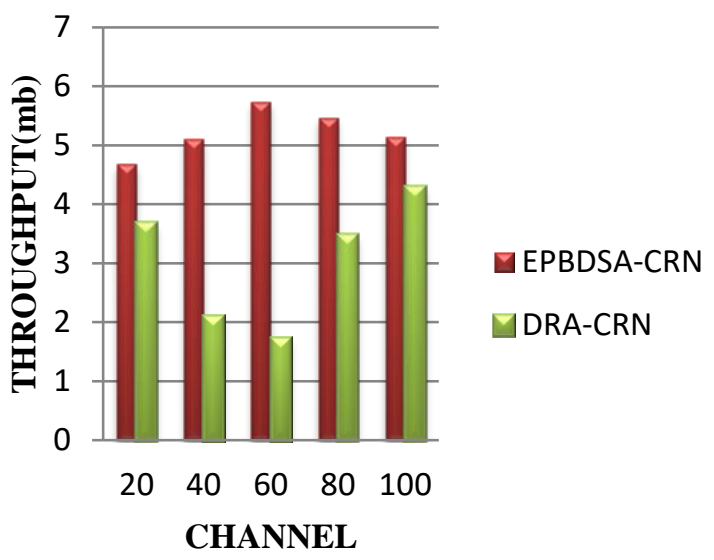

Figure.4 Channels vs. throughput

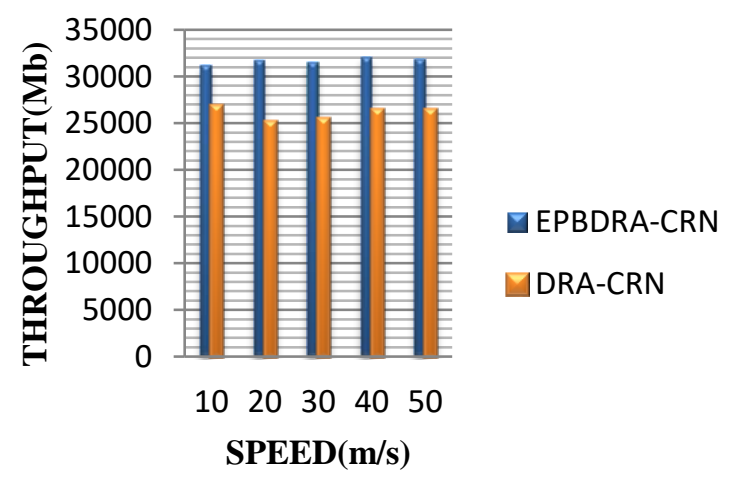

Figure.5 Speed vs. throughput

In Fig. 3, the proposed system is found to practice less delay since each SU is prioritized in accessing the idle channel without any interference occurring at the channel. Since each SU senses the channel before accessing the channel and also periodically checks for the existence of the PU, the interference introduced due to the sudden appearance of PU is reduced. Prioritization of SU's at the SBS leads to idle channel allocation robustly.

In Figs. 4 and 5 throughput in proposed system is $41 \%$ more efficient with changing the number of

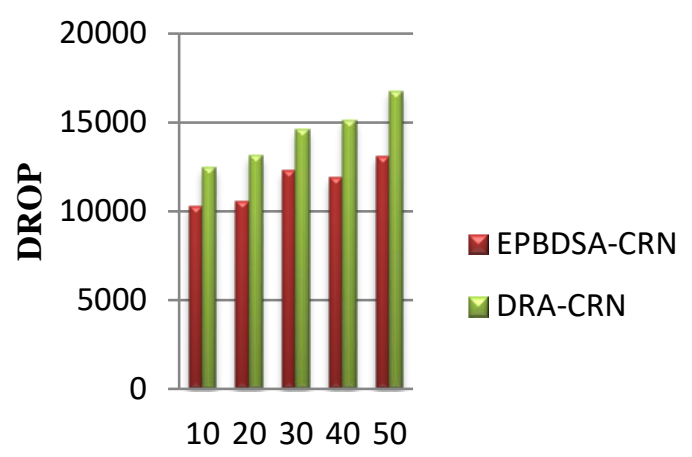

$\operatorname{SPEED}(\mathrm{m} / \mathrm{s})$

Figure.6 Speed vs. Drop

idle channels from 20 channels to 100 channels in the given network. With variations in the mobility of nodes from $10 \mathrm{~m} / \mathrm{s}$ to $50 \mathrm{~m} / \mathrm{s}$ the throughput of proposed system is found to be $3 \%$ efficient in comparison to existing system.

\section{Conclusion}

In this thesis, we could have understood scarcity of dynamic resource allocation and extended more knowledge about MIMO cognitive radio knowledge with dynamic data transmission carried between PU and SU. The PU transmits through SUs to destination so that the required nodes in $\mathrm{SN}$ are selected with specific constraints like MGR-SU, MDR-SU, MDRG-SU, and BES-SU. Our proposed EPBDRA-CRN system achieves a better performance with the smart antennas and transmission channels selection approach which satisfies the intellectual properties of networks like fairness and delay constraints. Nodes which are in neighborhood in the given network should operate on distinct accessible channels or cooperatively for many-to-many virtual array for MIMO communication on the same channel as per priority scheduling method. The performance result shows that our proposed algorithm is much more efficient in coordinating transmission in a MIMO-CRN. In simulation result, EPBDRA-CRN has been found to have better throughput than DRA-CRN. Our proposed algorithm minimized the interference at PU. Hence the performance of the entire network has improved better. The proposed algorithm achieves more than $27 \%$ efficiency compared to existing DRA-CRN. In future we can hybrid CR and MIMO technique with priority scheduling algorithm to reach best performance in forthcoming wireless communication system. 


\section{References}

[1] S. Senthilmurugan, J. Ansari, M. Petri, T.G. Venkatesh, and M. Petrova, "Channel Selection Algorithm for Cognitive Radio Networks with Heavy-Tailed Idle Times", arXiv: 1607.04450v1 [cs.N1], pp.1-4, 2016.

[2] S. Chu, X. Wang, and Y. Yang, "Adaptive Scheduling in MIMO-Based Heterogeneous AdHoc Networks", IEEE Transactions on Mobile Computing, Vol.13, No.5, pp.964-978, 2014.

[3] S.J. Kim and G.B. Giannakis, "Optimal Resource Allocation for MIMO AdHoc Cognitive Radio Networks", IEEE Transactions on Information Theory, Vol.57, No.5, pp.31173131, 2011.

[4] D.W.K. Ng, E.S. Lo, and R. Schober, "Dynamic Resource Allocation in MIMO-OFDMA Systems with Full-Duplex and Hybrid Relaying", IEEE Transaction on Communications, Vol.60, No.5, pp.1291-1304, 2012.

[5] A. Ghosh and W. Hamouda, "Cross-Layer Antenna Selection and Channel Allocation for MIMO Cognitive Radios", IEEE Transaction on Wireless Communications, Vol.10, No.11, pp. 3666-3674, 2011.

[6] X. Xiao, X. Tao, and J. Lu, "Energy-Efficient Resource Allocation in LTE-Based MIMOOFDMA Systems with User Rate Constraints", IEEE Transactions on Vehicular Technology, Vol.64, No.1, pp.185-197, 2015.

[7] Y. An, X. Huang, K. Zhu, and Y. Xiao, "Multiusers Cooperative Transmitting Algorithm in Cognitive WLAN", Journal of Networks, Vol.7, No.8, pp.1164-1169, 2012.

[8] M.G. Adian and M.G. Adyan, "Optimal and Suboptimal Resource Allocation in MIMO Cooperative Cognitive Radio Networks", Journal of Optimization, Article-ID: 190196, pp.1-14, 2014.

[9] N. Tadayon and S. Aissa, "A Multi-Channel Spectrum Sensing Fusion Mechanism for Cognitive Radio Networks: Design and Application to IEEE 802.22 WRANs", IEEE Transactions on Cognitive Communications and Networking, pp.1-13, 2016.

[10] Z. Khan, J.J. Lehtomaki, L.A. DaSilva, E. Hossain, and M.L. Aho, "Opportunistic Channel Selection by Cognitive Wireless Nodes under Imperfect Observations and Limited Memory: A Repeated Game Model", IEEE Transactions on Mobile Computing, Vol.15, No.1, pp.173-187, 2016.
[11] D.T. Hoang, D. Niyato, P. Wang, and D.I. Kim, "Opportunistic Channel Access and RF Energy Harvesting in Cognitive Radio Networks", IEEE Journal on Selected Areas in Communications, Vol.32, No.11, pp.2039-2052, 2014.

[12] R. Xie, F.R. Yu, and H. Ji, "Dynamic Resource Allocation for Heterogeneous Services in Cognitive Radio Networks With Imperfect Channel Sensing", IEEE Transactions on Vehicular Technology, Vol.61, No.2, pp.770780, 2012.

[13] C. Gao, S. Chu, X. Wang, "Distributed Scheduling in MIMO Empowered Cognitive Radio Ad Hoc Networks", IEEE Transactions on Mobile Computing, Vol.13, No.7, pp.14561458, 2014.

[14] B.S. Awoyemi, B.T.J. Maharaj, and A.S. Alfa, "Solving resource allocation problems in cognitive radio networks: a survey", EURASIP Journal on Wireless Communications and Networking, Vol.176, pp.1-14, 2016.

[15] S. Tapaswi, R.S. Jadoan, and P. Sharma, "Dynamic Spectrum Allocation Technique with Reduced Noise in Cognitive Radio Networks", International Journal of Computer Science and Network Security, Vol.10, No.1, pp.274-280, 2010.

[16] W. Wang, K.G. Shin, and W. Wang, "Distributed Resource allocation Based on Queue Balancing in Multihop Cognitive Radio Networks", IEEE/ACM Transactions on Networking, Vol.20, No.3, pp.837-850, 2012.

[17] A. Mohamedou, A. Sali, B. Ali, and M. Othman, "Dynamical Spectrum Sharing and Medium Access Control for Heterogeneous Cognitive Radio Networks", International Journal of Distributed Sensor Networks, Vol.2016, pp.1-15, 2016.

[18] X. Gelabert, O. Sallent, J.P. Romero, and R. Agusti, "Spectrum sharing in cognitive radio networks with imperfect sensing: A discreatetime Markov model", Computer Networks, Vol.54, pp.2919-2536, 2010.

[19] M. Min, Y.S. Jeon, and G.H. Im, "On Achievable Rate of User Selection for MIMO Broadcast Channels with Limited Feedback", IEEE Transactions on Communications, Vol.65, No.1, pp.122-135, 2017.

[20] M. Khasawneh, S. Alrabaee, A. Agarwal, N. Goel, and M. Zaman, "Power trading in cognitive radio networks", Journal of Network and Computer Applications, 2016.

[21] P. Reba, G.U. Maheswari, and M.S. Babu, "Multiple Antenna Selection for underlay 
cognitive radio system with interference constraint", Wireless Pers Commun, springer publication 2017.

[22] S. Kumar, A. Sarkar, and A. Sur, "A Resource Allocation Framework for Adaptive Streaming over LTE", Journal of Network and Computer Application, Vol. 97, pp. 126-139, 2017.

[23] P.M.R.D. Santos, M.A. Kalil, O. Artemenko, A. Lavrenko, and A.M. Tjel, "Self-Organized Common Control Channel Design for Cognitive Radio Ad Hoc Networks", In: Proc. of 2013 IEEE 24th International Symposium on Personal, Indoor and Mobile Radio Communications: Mobile and Wireless Networks, pp. 2419-2423, 2013

[24] C.K. Tan, T.C. Chuah, and S.W. Tan, "Resource allocation for OFDMA-based multicast cognitive radio networks using a Stackelberg pricing game", Computer Communications, Vol. 88, pp. 57-72, 2016.

[25] C. Lucas- Estañ and J. Gozalvez, "Distributed Radio Resource allocation for Device-to-Device Communications Underlying Cellular Networks", Journal of Network and Computer Applications, Vol. 99, pp. 120-130, 2017.

[26] S. Tamilarasan and K. Parasuraman, "Dynamic Resource Allocation and Priority Based Scheduling for Heterogeneous Services in Cognitive Radio Networks", International Journal of Intelligent Engineering and Systems, Vol.9, No.3, pp. 127-137, 2016.

[27] S. Tamilarasan and K. Parasuraman, "Appropriate Channel Selection for Dynamic Resource Allocation with Priority Scheduling Approach in Multi-hop Cognitive Radio Networks", International Journal of Intelligent Engineering and Systems, Vol.10, No.2, pp. 110, 2017. 